\title{
Editorial:
}

\section{Intelligent unmanned systems: important achievements and applications of new generation artificial intelligence}

\author{
Cheng $\mathrm{WU}^{1,2}$, Tao ZHANG ${ }^{\dagger 1,2}$ \\ ${ }^{I}$ Department of Automation, Tsinghua University, Beijing 100084, China \\ ${ }^{2}$ Beijing National Research Center for Information Science and Technology, Beijing 100084, China \\ †E-mail: taozhang@tsinghua.edu.cn
}

https://doi.org/10.1631/FITEE.2030000

The advancement of science and technology is an important indicator of a nation's overall strength. More recently, with the rise of new generation artificial intelligence (AI), we are facing a new industrial revolution. For example, the development of intelligent unmanned systems (IUSs) will soon become a landmark achievement for AI development. In the next 3-5 years, applications of intelligent robots (service, industry, etc.) in China will have a wide range of advancements. In particular, unmanned aerial vehicles (UAVs) will attain multi-industry, largescale applications. The autopilot system will soon complete a demonstration project. This means China will develop the core common technology of rail transit autonomous driving. Significant progress will be made in intelligent workshops and factories, and a set of Chinese standards in line with international standards will be formed.

IUS is an artificial system that can be operated or managed using advanced technology without any human intervention. Over the years, humans have created various types of unmanned systems. More importantly, as human knowledge develops, the technology level of unmanned systems is gradually improved. IUS is also a complex system, which is composed of many technologies, such as machinery, control, computer, communication, and material. AI

\footnotetext{
${ }^{\ddagger}$ Corresponding author

(7) ORCID: Tao ZHANG, https://orcid.org/0000-0002-2980-6281

(C) Zhejiang University and Springer-Verlag GmbH Germany, part of Springer Nature 2020
}

is undoubtedly one of the key technologies needed to develop IUSs. Autonomy and intelligence are the two most important characteristics of IUSs. The most effective way to achieve and continuously improve these two characteristics is to use various technologies of $\mathrm{AI}$, such as intelligent perception (image, voice recognition, etc.), human-computer interaction, intelligent decision-making, learning, and reasoning.

Unmanned systems are a variety of systems developed to work without human inputs, but the highest level of "unmanned" systems is man-machine integration. Specifically, human-computer integration means that human beings connect their nervous system with computers and other machines to make up for the defects of human senses and movement, and to determine the integration of human consciousness and computer AI. The combination of AI and unmanned systems is expected to develop technologies that can alter life, so as to strengthen human function (especially the disabled and the elderly) and to improve the quality of human life. Generally, humancomputer integration includes human-computer cooperation. The relationship between human and machine is no longer a master-slave relationship, or an alternative relationship, but a partnership. Efficiency and flexibility can be improved, if people control multiple unmanned systems to work together; the coordination and interaction between people and unmanned systems will significantly improve different aspects of the capabilities of the systems. However, for a considerable part of labor-intensive 
work, the unmanned system may not be efficient. Therefore, IUSs will be an important embodiment of man-machine integration.

Compared with the traditional unmanned system, the IUS has more potential to applications. The emergence of various types of IUSs will have a significant impact on human life and society. At present, IUSs include mainly autonomous driving vehicles, UAVs, service-oriented robots, intelligent industrial robots, space robots, marine robots, and unmanned workshop/intelligent factories.

The new generation AI development plan released by China captures "intelligent technology of autonomous unmanned systems." It focuses on breaking through common technologies such as computing architecture of autonomous unmanned system, perception and understanding of complex dynamic scene, real-time precise positioning, adaptive intelligent navigation for complex environment, autonomous control of UAV, intelligent technologies including self-driving automobile, ship and rail transit, and core technologies (such as service robot and special robot), supporting the application and industrial development of unmanned systems.

In this context, the Chinese Academy of Engineering organized a special issue on IUSs in Frontiers of Information Technology \& Electronic Engineering. The types of IUSs examined in this special issue include robotic exoskeleton, intelligent ground vehicles, underwater vehicles, and UAVs. After rigorous review processes, 10 papers have been selected for this special issue, including two review articles, one tutorial, and seven research articles.

With an increasing demand for reusable launchers and more scientific returns from space exploration, pinpoint soft landing has become a basic requirement. Zheng-yu SONG and his collaborators summarize the autonomous guidance methods for pinpoint soft landing on celestial surfaces. They achieve this by comparing the characteristics of three scenarios for landing on the Moon, Mars, and Earth. The constraints and performance index functions for 3- and 6-degree-of-freedom landing problems are summarized. In addition, a general problem description is formed, and three classes of methods, including analytical algorithms, numerical optimization algorithms, and learning-based methods, are discussed in detail.
With the development of sensor fusion technologies, there has been much research on intelligent ground vehicles, where obstacle detection is one of the major aspects of vehicle driving. Quan PAN and his collaborators summarize the main considerations for on-board multi-sensor configuration of intelligent ground vehicles in the off-road environment, and provide users with a guideline for selecting sensors based on their performance requirements and application environments. State-of-the-art multi-sensor fusion methods and system prototypes are reviewed, which are associated with heterogeneous sensor configurations.

The rapid growth of e-commerce, constantly rising labor cost, and increasingly demanding expectation of customers have prompted warehouses worldwide to be equipped with newer and more advanced automation technologies. It is critical that warehouse automation technologies be unprecedentedly efficient, adaptive, scalable, and fault-tolerant. Kai CAI presents a new cyber-physical control method that achieves safe, deadlock-free, efficient, and adaptive behavior of multiple robots serving goods-to-man logistic operations.

The wearable exoskeleton is a powered anthropomorphic electromechanical system for strength enhancement, load carrying, exercise rehabilitation, and walking assistance. More importantly, accurate acceleration acquisition is a critical issue in the design of a robotic exoskeleton system. Tao ZHANG and his collaborators present a fixed-time constrained acceleration reconstruction scheme for robotic exoskeleton through a neural network. The proposed scheme can achieve high-performance observation and provide high-precision acceleration estimate in the actual exoskeleton system. These novel properties will help develop better acceleration-dependent control algorithms.

The lower-limb-assisted exoskeletons are widely researched to achieve movement assistance or rehabilitation training. Although some cable-driven prototypes are being developed, most of the existing cable-driven prototypes can only assist unidirectional movement due to compliance and light weight. Tian-miao WANG and his collaborators present an untethered cable-driven ankle exoskeleton that can achieve plantarflexion-dorsiflexion bidirectional 
motion using a pair of single motors.

Wan-ying RUAN and Hai-bin DUAN present a multi-objective social learning pigeon-inspired optimization (MSLPIO) method, which is applied to avoid obstacles for UAV formation. MSLPIO has better convergence performance compared to the improved multi-objective pigeon-inspired optimization and the improved non-dominated sorting genetic algorithm (NSGA-II).

The deepest ocean depth so far reported is about $11000 \mathrm{~m}$, in the Mariana Trench in the Western Pacific Ocean. The hybrid unmanned underwater vehicle, Haidou, was developed to perform scientific surveys at the deepest parts of the Earth's oceans. The maximum depth recorded by Haidou was 10905 m, and it was to determine the precise location of the deepest point. Chuan-xu CHEN and his collaborators present a terrain matching method to overcome the localization problem without using an acoustic positioning system at the deepest depth of the Earth's oceans, so that the Haidou hybrid underwater vehicle becomes one of the few vehicles that can dive the full-ocean depth.

Point set registration has been a topic of significant research interest in the field of mobile IUSs. Wen-jie ZHAO and his collaborators present a novel three-dimensional point set registration method based on a new type of map representation, called the Gaussian process map. The proposed method can be adopted for a complete simultaneous localization and mapping (SLAM) framework.

Flight control systems are critical elements in a variety of missions and applications for UAVs. Huan $\mathrm{HU}$ and Qing-ling WANG present a proximal policy optimization with integral compensator (PPO-IC) algorithm for the development of the UAV intelligent controller, which effectively reduces the steady-state error in speed tracking, and significantly improves the tracking accuracy.

Coordinating multiple UAVs is a difficult technique in highly dynamic and sophisticated environments. Zhi-feng ZHAO and his collaborators present a strategy for multi-UAVs to acquire targets with limited prior knowledge, based on digital pheromones and current mainstream unmanned system controlling algorithms. This method can yield superior performance in terms of coverage, detection and revisit efficiency, and the capability to avoid obstacles.

The papers included in this special issue cover a broad spectrum of current research topics on IUSs, such as autonomous guidance, map reconstruction, obstacle detection, localization, control, and multiple system collaboration. We hope these papers will be beneficial to readers in IUSs and related fields.

Finally, we would like to show our special thanks to the authors and reviewers, for their support and valuable contributions to this issue. We appreciate the work of the editorial staff, and the Editors-inChief, Profs. Yun-he PAN and Xi-cheng LU.

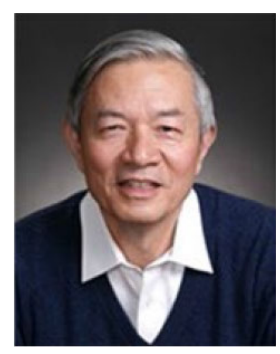

Prof. Cheng WU received his BS and MS degrees in Electrical Engineering from Tsinghua University, Beijing, China, in 1962 and 1966, respectively. He was a postdoctoral fellow at Case Western Reserve University, USA from 1981 to 1983 . He is Director of the State CIMS Engineering Research Center. He is an Academician of the Chinese Academy of Engineering. His current research interests include computer-integrated manufacturing system, complex system modeling, scheduling and planning, networked manufacturing, among others.

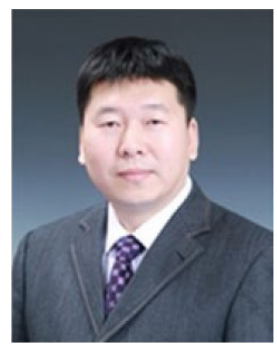

Prof. Tao ZHANG received his BS, $\mathrm{MS}$, and $\mathrm{PhD}$ degrees in Electrical Engineering from Tsinghua University, Beijing, China, in 1993, 1995, and 1999, respectively. He received his second $\mathrm{PhD}$ degree in Electrical Engineering from Saga University, Saga, Japan, in 2002. Now, he is a professor and Vice Dean of the School of Information Science and Technology, Head of the Department of Automation, Tsinghua University. He is an IET fellow, IEEE senior member, member of the IFAC Technical Committee of Robotics, AIAA member, and technical editor of IEEE/ASME Transactions on Mechatronics. His current research interests include robotics, AI, control theory, among others. 